\title{
APPLICATION OF THE MEDIATION ANALYSIS APPROACH TO CANCER PREVENTION TRIALS RELATING TO CANCER SEVERITY
}

\author{
Yasutaka Chiba \\ Clinical Research Center, Kinki University Hospital, Osaka, Japan
}

Received 2014-09-08; Revised 2014-10-06; Accepted 2014-10-11

\begin{abstract}
In a cancer prevention trial, an outcome such as cancer severity cannot be evaluated in individuals who do not develop cancer. In such a situation, the principal stratification approach has been applied. Under this approach, the Principal Strata Effect (PSE) has been considered, which is defined as the effect of treatment on the outcome among the subpopulation in which individuals would have developed cancer under either treatment arm. However, in this study, the author does not apply this approach to the situation. Instead, the author discusses the mediation analysis approach, in which Natural Direct and Indirect Effects (NDE and NIE) are considered. This approach has an advantage as it considers two possible mechanisms of treatment control of cancer severity: The first is that the treatment may prevent an individual from getting cancer, which could be regarded as control of cancer severity; the second is that even if the treatment does not prevent an individual from getting cancer, it may still impair the cancer severity. The former mechanism corresponds to the NIE and the latter corresponds to the NDE, although the PSE can consider only the latter mechanism. Methodologies proposed in the context of vaccine trials are applied to data from a randomized prostate cancer prevention trial.
\end{abstract}

Keywords: Natural Direct Effect, Natural Indirect Effect, Principal Strata Effect, Sensitivity Analysis

\section{INTRODUCTION}

A multi-center, double-blind, randomized prostate cancer prevention trial studied the effects of finasteride on the prevalence of prostate cancer in healthy males screened over a period of 7 years (Thompson et al., 2003). In total, 18,882 males aged 55 years or older with no history or indicators of prostate cancer were randomized to receive either 5 $\mathrm{mg}$ of finasteride per day or placebo. At each annual follow-up examination, participants with a prostatespecific antigen level exceeding a threshold of $3 \mathrm{ng} \mathrm{mL}^{-1}$ or with an abnormal digital rectal examination were referred for a prostate biopsy. Additionally, all participants who had not been diagnosed with prostate cancer during the trial underwent an end-of-study prostate biopsy at their seventh and final visit. Due to early closure of the study, 15,990 of the 18,882 males were assessable for the endpoint.

Of the 15,990 males, the cancer status of the 10,182 males was identified by biopsy. The cancer status and severity for 5,808 (15,990-10,182) males was unknown due to missing data. These missing data would be non-random and thus the results that would be obtained if their cancer status and severity had been observed were estimated (Redman et al., 2008) by applying the inverse probability of censoring weighted method (Robins et al., 1992). The observed and estimated results are summarized in Table 1 (Redman et al., 2008). In this study, we use the estimated result in the following analyses without taking error by estimation into account, because we cannot access the raw data and therefore cannot use the information. 
Yasutaka Chiba / American Journal of Biostatistics 4 (2): 45-51, 2014

Table 1. Observed and estimated numbers of prostate cancer detected on biopsy (Redman et al., 2008).

\begin{tabular}{llc}
\hline & Finasteride arm & Placebo arm \\
& 7,966 & 8,024 \\
\hline Prostate cancer & & \\
Estimate of overall prevalence & $1,171(14.7 \%)$ & $1,693(21.1 \%)$ \\
Observed & $823(16.6 \%)$ & $1,194(22.9 \%)$ \\
High-grade cancer & & $337(4.2 \%)$ \\
Estimate of overall prevalence & $382(4.8 \%)$ & $252(4.8 \%)$ \\
Observed & $288(5.8 \%)$ & \\
\hline
\end{tabular}

The estimated result showed that prostate cancer was detected in $1,171(14.7 \%)$ of the 7,966 males in the finasteride arm and in $1,693(21.1 \%)$ of the 8,024 males in the placebo arm. This suggests that finasteride lowered the risk for prostate cancer, where the risk difference was $-6.4 \% \quad(95 \%$ confidence interval $[\mathrm{CI}]:-7.6 \%,-5.2 \%$; $\mathrm{p}<0.001)$. However, males with high-grade prostate cancer, which was defined as a Gleason score (Gleason, 1966) $\geq 7$, were $382(4.8 \%)$ in the finasteride arm and $337(4.2 \%)$ in the placebo arm. The risk difference was $0.6 \%$ (95\% CI: $-0.0 \%, 1.2 \%$; $p=$ 0.069 ), suggesting that the risk was slightly higher in the finasteride arm than in the placebo arm, but finasteride might not control cancer severity.

The analysis can involve dividing the above total effect of finasteride on cancer severity into two mechanisms; one is that finasteride may prevent an individual from developing cancer and thereby control his cancer severity even if cancer develops and the other is that even if finasteride does not prevent an individual from developing cancer, it may still affect cancer severity. In the context of the mediation analysis approach (Pearl, 2001; Robins, 2003; van der Laan and Petersen, 2008; Shpitser and VanderWeele, 2011), the former mechanism corresponds to the Natural Indirect Effect (NIE) and the latter to the Natural Direct Effect (NDE).

In prevention trials such as this trial, the principal stratification approach (Frangakis and Rubin, 2002; Rubin, 2004; Shepherd et al., 2008; Chiba, 2012) has been discussed. However, this approach has the disadvantage that it does not consider the former mechanism that finasteride may prevent an individual from developing cancer, which could be regarded as control of cancer severity. In this study, we apply the mediation analysis approach to the prostate cancer prevention trial, using methodologies proposed in the context of vaccine trials (VanderWeele and Tchetgen Tchetgen, 2011; VanderWeele et al., 2012; Chiba 2013; Chiba and Taguri, 2013) that allow this mechanism to be considered.

\section{MATERIALS AND METHODS}

\subsection{Notation and Definition}

Throughout this study, we use the following notation for the observed variables. $A$ denotes the assigned treatment, where $A=1$ if a participant was in the finasteride arm and $A=0$ if in the placebo arm. $M$ denotes whether the biopsy detected prostate cancer; $M=1$ if a participant developed prostate cancer and $M$ $=0$ if no cancer was detected. $Y$ denotes cancer severity; $Y=1$ if high-grade cancer (the Gleason score $\geq 7$ ) and $Y=0$ if not high-grade cancer (the Gleason score $\leq 6$ ). Because a participant could not have highgrade cancer severity when he did not develop the cancer, the value of $Y$ cannot be 1 when $M=0$; i.e., $\operatorname{Pr}(Y=1 \mid A=a, M=0)=0$.

For each participant, it is also possible to consider the potential outcomes (Rubin, 1974, 1978, 1990), which correspond to the outcomes of the participant had he been in the other arm of the trial. $M(a)$ denotes the cancer status for each participant under $A=a$. Likewise, $Y(a)$ denotes the cancer severity for each participant under $A=a$. Due to randomization of treatment, the potential outcomes $Y(a)$ and $M(a)$ are independent of the treatment $A$. This independency implies $\operatorname{Pr}(Y(a)=y)=\operatorname{Pr}(Y(a)=y \mid A$ $=a)$ and $\operatorname{Pr}(M(a)=m)=\operatorname{Pr}(M(a)=m \mid A=a)$.

We assume that $Y(a)$ and $M(a)$ for an individual do not depend on the treatment status of other individuals. This assumption is sometimes referred to as the nointerference assumption (Cole and Hénan, 2008). We further require the consistency assumption (VanderWeele, 2009) that $Y(A)=Y$ and $M(A)=M$ for all individuals, so the respective values of $Y$ and $M$ that would have been observed if $A$ had been set to what in fact they were are equal to the values of $Y$ and $M$ that were observed. This assumption implies that $\operatorname{Pr}(Y(a)=$ $1 \mid A=a)=\operatorname{Pr}(Y=1 \mid A=a)$, that $\operatorname{Pr}(M(a)=1 \mid A=a)=$ $\operatorname{Pr}(M=1 \mid A=a)$ and that $\operatorname{Pr}(Y(a)=1 \mid A=a, M=m)=$ $\operatorname{Pr}(Y=1 \mid A=a, M=m)$. 


\subsection{Natural Direct and Indirect Effects}

Suppose that, in addition to potentially intervening to assign the treatment randomly, we could, at least hypothetically, intervene to develop or not develop cancer. Then, $Y(a, m)$ would denote cancer severity if we set the treatment to $a$ and the cancer status to $m$. This potential outcome is used to define the NDE and NIE. The NDE is defined as follows:

$$
\text { NDE: }=\operatorname{Pr}(Y(1, M(1))=1)-\operatorname{Pr}(Y(0, M(1))=1)
$$

This compares the potential cancer severity if the participant had received finasteride versus placebo and he had the cancer status as if he had received finasteride. If Equation 1 is non-zero, this will be because even when the participant received finasteride and developed cancer, finasteride itself affects cancer severity.

The NIE is defined as follows:

$$
\text { NIE: }=\operatorname{Pr}(Y(0, M(1))=1)-\operatorname{Pr}(Y(0, M(0))=1)
$$

The term $Y(0, M(1))$ considers what the potential cancer severity is if the participant receives placebo, but we set his cancer status to the status that it would have been if he received finasteride. Equation 2 compares this potential outcome to $Y(0, M(0))$, which is the potential cancer severity if the participant receives placebo and we set the cancer status to the status that it would have been if the participant received the placebo. For Equation 2 to be non-zero, $M(1)$ and $M(0)$ have to differ; i.e., finasteride would have to affect the cancer status and that change in cancer status would have to change the cancer severity, even if the participant had received placebo. Essentially, Equation 2 is non-zero if the finasteride prevents an individual from cancer and that in turn controls cancer severity.

The term $Y(a, M(a))$ is equal to $Y(a)$ and thus we can decompose the total effect $\operatorname{Pr}(Y(1)=1)-\operatorname{Pr}(Y(0)=1)$ into the NDE and NIE as follows:

$$
\begin{aligned}
& \operatorname{Pr}(Y(1)=1)-\operatorname{Pr}(Y(0)=1) \\
& =\operatorname{Pr}(Y(1, M(1))=1)-\operatorname{Pr}(Y(0, M(0))=1) \\
& =\operatorname{Pr}(Y(1, M(1))=1)-\operatorname{Pr}(Y(0, M(1))=1) \\
& +\operatorname{Pr}(Y(0, M(1))=1)-\operatorname{Pr}(Y(0, M(0))=1) \\
& =\mathrm{NDE}+\mathrm{NIE}
\end{aligned}
$$

\subsection{Sensitivity Analysis}

The NDE and NIE can be estimated with identifying assumptions (Lange et al., 2012; Valeri and VanderWeele, 2013), if all covariates between cancer status and severity are observed. However, unfortunately, we do not have the information for covariates. Therefore, we implement a sensitivity analysis under the following assumption:

\section{Assumption 1}

$M(1) \leq M(0)$ for all individuals.

This assumption is sometimes referred to as the monotonicity assumption (Manski, 1997) and implies that $\operatorname{Pr}(M(1)=1, M(0)=0)=0$; i.e., there is no individual that he would develop cancer if he received finasteride but would not develop if he received placebo. Thus, Assumption 1 assumes that fnasteride prevents developing prostate cancer and it would be reasonable in this trial.

When $\operatorname{Pr}(Y=1 \mid A=a, M=0)=0$, under Assumption $1, \operatorname{Pr}(Y(a, M(1))=1)$ can be expressed as follows:

$$
\begin{aligned}
& \operatorname{Pr}(Y(a, M(1))=1) \\
& =\operatorname{Pr}(Y(a)=1 \mid A=1, M=1) \operatorname{Pr}(M=1 \mid A=1)
\end{aligned}
$$

The derivation is found elsewhere (Chiba, 2013; Chiba and Taguri, 2013). Using Equation 4, Equation 1 can be expressed as:

$$
\begin{aligned}
& \mathrm{NDE}=\{\operatorname{Pr}(Y(1)=1 \mid A=1, M=1) \\
& -\operatorname{Pr}(Y(0)=1 \mid A=1, M=1)\} \\
& \times \operatorname{Pr}(M=1 \mid A=1)
\end{aligned}
$$

Using Equation 3, the NIE is given by subtracting Equation 5 from the total effect $\operatorname{Pr}(Y(1)=1)-\operatorname{Pr}(Y(0)=$ 1). Note that

$$
\begin{aligned}
& \operatorname{Pr}(Y(1)=1 \mid A=1, M=1)-\operatorname{Pr}(Y(0)=1 \mid A=1, M=1) \\
& =\operatorname{Pr}(Y(1)=1 \mid M(1)=M(0)=1) \\
& -\operatorname{Pr}(Y(0)=1 \mid M(1)=M(0)=1)
\end{aligned}
$$

Which holds under Assumption 1, is the Principal Strata Effect (PSE) that is a causal effect for the population in which participant would develop cancer regardless of the assigned treatment.

Here, we set the sensitivity parameter as: 
$\alpha=\operatorname{Pr}(Y(0)=1 \mid A=1, M=1)$

$-\operatorname{Pr}(Y(0)=1 \mid A=0, M=1)$

Which is proposed for a sensitivity analysis of the PSE (Chiba, 2010; Chiba and VanderWeele, 2011). The sensitivity parameter is the difference between the cancer severity that would have been observed if the participant received placebo when comparing the two different populations: The first is that in which the participant received finasteride $(A=1)$ and the second is that in which participant received placebo $(A=0)$, where the cancer status is equal in these two populations $(M=1)$. The interpretation of $\alpha$ is then simply the difference of the expected cancer severity under placebo for these two populations.

Using Equation 6, Equation 5 can be expressed as:

NDE

$=\operatorname{Pr}(Y=1 \mid A=1)$

$-\{\operatorname{Pr}(Y=1 \mid A=0, M=1)+\alpha\} \operatorname{Pr}(M=1 \mid A=1)$

$=\{\operatorname{Pr}(Y=1 \mid A=1, M=1)-\operatorname{Pr}(Y=1 \mid A=0, M=1)\}$

$\times \operatorname{Pr}(M=1 \mid A=1)-\alpha \operatorname{Pr}(M=1 \mid A=1)$

And the NIE can be expressed as:

NIE

$=\{\operatorname{Pr}(Y=1 \mid A=0, M=1)+\alpha\} \operatorname{Pr}(M=1 \mid A=1)$

$-\operatorname{Pr}(Y=1 \mid A=0)$

$=\{\operatorname{Pr}(M=1 \mid A=1)-\operatorname{Pr}(M=1 \mid A=0)\}$

$\times \operatorname{Pr}(Y=1 \mid A=0, M=1)+\alpha \operatorname{Pr}(M=1 \mid A=1)$

where, $\operatorname{Pr}(Y=1 \mid A=a, M=0)=0$ was applied.

Using Equations 7 and 8, a sensitivity analysis can be conducted as follows. The sensitivity parameter $\alpha$ is set by the researcher according to what is considered plausible. The parameter can be varied over a range of plausible values to examine how conclusions vary according to differences in parameter values. The results of the sensitivity analysis can be displayed graphically, where the horizontal axis represents the value of the sensitivity parameter and the vertical axis represents the values of the NDE and NIE.

\subsection{Bounds for the Sensitivity Parameter}

In some situations, it may be troublesome for researchers to determine a range of $\alpha$ to examine. Therefore, we here introduce the bounds for $\alpha$.
Initially, we present the bounds only under Assumption 1 , which is sometimes referred to as the large sample bounds (Zhang and Rubin, 2003; Chiba, 2012). In Equation 6, because $\operatorname{Pr}(Y(0)=1 \mid A=0, M=1)=\operatorname{Pr}(Y=1 \mid A=0, M=$ 1) can be identified, we need to derive the bounds for $\operatorname{Pr}(Y(0)=1 \mid A=1, M=1)=\operatorname{Pr}(Y(0)=1 \mid M(1)=M(0)=1)$, which holds under Assumption 1. Because $\operatorname{Pr}(M(1)=1$, $M(0)=1)=\operatorname{Pr}(M=1 \mid A=1)$ under Assumption 1, of participants with $(M(1), M(0))=(1,1)$, the number of those who were assigned to the placebo arm is expected to be:

$N^{*}:=N \operatorname{Pr}(A=0) \operatorname{Pr}(M=1 \mid A=1)$

where, $N$ is the total number of participants and $N \operatorname{Pr}(A$ $=0)$ is the number of participants who were assigned to the placebo arm. Using this expected number, the lower bound of $\operatorname{Pr}(Y(0)=1 \mid A=1, M=1)=\operatorname{Pr}(Y(0)=$ $1 \mid M(1)=M(0)=1)$ is:

$\left\{\begin{array}{cl}\frac{N^{*} \times 0}{N^{*}} & \text { if } N^{*} \leq N_{010} \\ \frac{N_{011} \times 0+\left(N^{*}-N_{010}\right) \times 1}{N^{*}} & \text { if } N^{*}>N_{010}\end{array}\right.$

where, $N_{a m y}=N \operatorname{Pr}(A=a, M=m, Y=y)$ is the number of participants with $(A, M, Y)=(a, m, y)$ and the upper bound is:

$\left\{\begin{array}{cl}\frac{N^{*} \times 1}{N^{*}} & \text { if } N^{*}<N_{011} \\ \frac{\left(N^{*}-N_{011}\right) \times 0+N_{011} \times 1}{N^{*}} & \text { if } N^{*} \geq N_{011}\end{array}\right.$

Equation 6 and 9-11 yield the following bounds for $\alpha$ after some algebra:

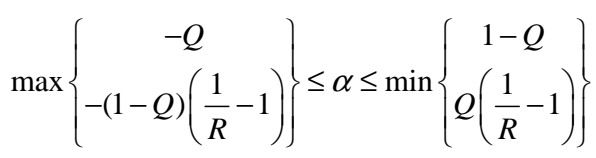

where, $Q=\operatorname{Pr}(Y=1 \mid A=0, M=1)$ and $R=\operatorname{Pr}(M=1 \mid A=$ 1) $\operatorname{Pr}(M=1 \mid A=0)$.

Next, we present bounds for $\alpha$ under Assumption 1 plus the following assumption, which is sometimes referred to as the assumption of ranked average score (Zhang and Rubin, 2003):

\section{Assumption 2}

$\operatorname{Pr}(Y(0)=1 \mid A=1, M=1) \geq \operatorname{Pr}(Y(0)=1 \mid A=0, M=1)$.

The first population in which the participant received finasteride and developed cancer $(A=1, M=1)$ would 
be less healthy than the second population in which the participant received placebo and developed cancer $(A=$ $0, M=1)$, because the first population is composed of participants who had high-grade cancer in spite of taking the treatment while some of participants in the second population might not have high-grade cancer if they had taken the treatment. Thus, under the scenario in which the participant receives placebo, the grade of cancer is likely to be higher in the first than in the second population and then Assumption 2 would be reasonable.

It is obvious that $\alpha \geq 0$ under Assumption 2 from Equation 6.

\section{RESULTS}

In this study, we implemented the sensitivity analysis for the NDE and NIE as presented in the section above.
Before the implementation, we determined the bounds for $\alpha$ to determine the range to be examined. Equation 12 yielded the bounds for $\alpha$ of $-0.199 \leq \alpha \leq 0.087$. By adding Assumption 2, the bounds were narrowed to $0 \leq \alpha \leq 0.087$. For this range of $\alpha$, we implemented the sensitivity analysis using Equation 7 and 8. The results are shown in Fig. 1 for the NDE and Fig. 2 for the NIE, where the variances of product terms in Equation 7 and 8 were calculated using the delta method; $\operatorname{var}(\hat{s} \hat{t})=$ $\operatorname{var}(\hat{s}) \operatorname{var}(\hat{t})+s^{2} \operatorname{var}(\hat{t})+t^{2} \operatorname{var}(\hat{s})$. The respective lower and upper limits of the NDE were $0.56 \%$ (95\% CI: $0.02 \%, 1.14 \%)$ and $1.83 \%$ (95\% CI: $1.27 \%, 2.40 \%)$ and those of the NIE were $-1.27 \%$ (95\% CI: $-1.82 \%,-0.73 \%)$ and $0.00 \%$ (95\% CI: $-0.57 \%, 0.57 \%)$. Note that the lower limit of the NDE is equal to the total effect and that the upper limit of the NIE is 0 from Equation 3.

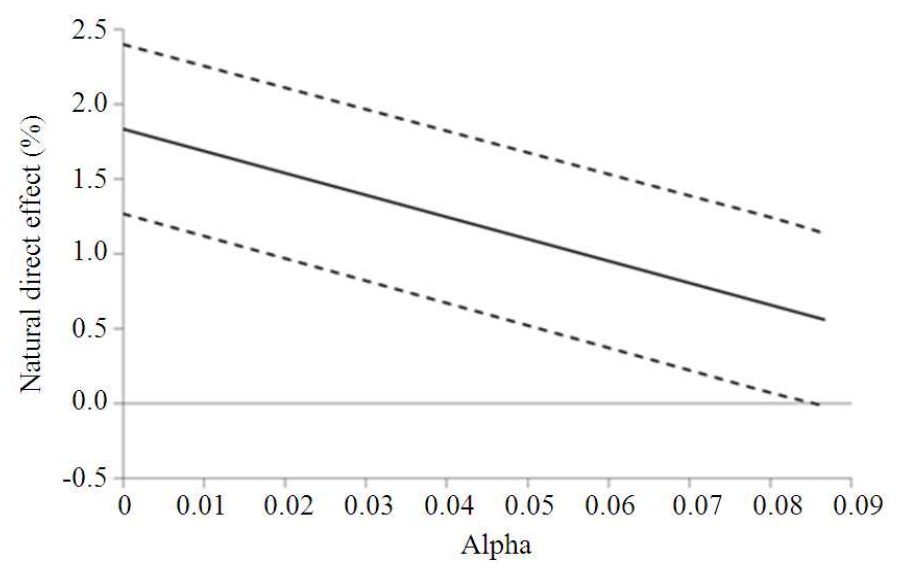

Fig. 1. Sensitivity analysis of the natural direct effect; the solid line indicates the natural direct effect and broken lines indicate $95 \%$ confidence interval

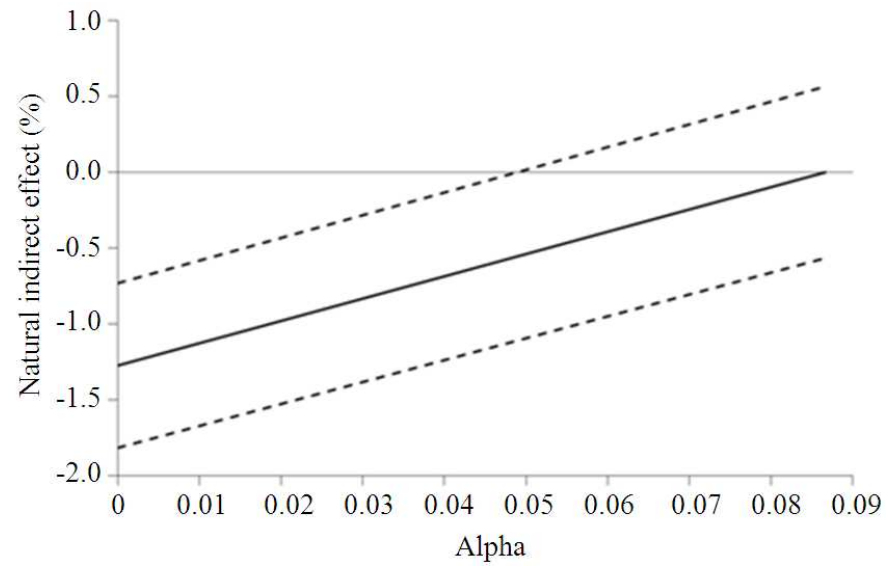

Fig. 2. Sensitivity analysis of the natural indirect effect; the solid line indicates the natural indirect effect and broken lines indicate $95 \%$ confidence interval 


\section{DISCUSSION}

Figure 2 shows that NIE $<0$ if the true value of $\alpha$ is small. This may be because finasteride only prevents indolent cancers that would never require treatment, as some prostate cancer researchers have argued (Phillips, 2008). If the argumentation is true, it is more reasonable to interpret as that finasteride only prevents an individual from indolent cancers and thereby the cancer severity remains low even if indolent cancer developed, rather than the interpretation mentioned in the introduction, that finasteride may prevent an individual from developing cancer and thereby control his cancer severity even if cancer develops.

However, NDE $>0$ as shown in Figure $\mathbf{1}$ and as the value of $\alpha$ is smaller, the estimate is larger. This makes interpretation of the results challenging, because finasteride promotes the growth of high-grade tumors directly and not through the prevention of cancer. The author does not have the answer to this problem. However, some research has found differential misclassification for grading of cancer severity, where the sensitivity is lower in the placebo arm than in finasteride arm (Thompson et al., 2006; Lucia et al., 2007). When such misclassification truly exists, if cancer severity in the placebo arm is measured with the same sensitivity as in the finasteride arm, $\operatorname{Pr}(Y=1 \mid A=0, M=$ 1) will be estimated as a larger value than presented in this study. The NDE will then be estimated as a smaller value, as seen from Equation 7. Similarly, the NIE will be estimated as a smaller value, as seen from Equation (8), where $\operatorname{Pr}(M=1 \mid A=1)-\operatorname{Pr}(M=1 \mid A=0)=$ $1171 / 7966-1693 / 8024=-6.4 \%<0$.

\section{CONCLUSION}

If differential misclassification for grading of cancer severity is truly exists, the NIE will be negative, although the absolute value may be small. However, it cannot be denied that finasteride only prevents indolent cancers that would never require treatment. It will not be affirmed that the NDE will be negative.

In a cancer prevention trial with an outcome such as cancer severity, which cannot be evaluated in individuals who do not develop cancer, we discussed the mediation analysis approach. As seen in the section above, this approach can lead to deeper discussion of the causal effects of treatment on an outcome such as cancer severity.

\section{ACKNOWLEDGEMENT}

This Study was supported partially by Grant-in-Aid for Scientific Research (No. 23700344) from the Ministry of Education, Culture, Sports, Science and Technology of Japan.

\section{REFERENCES}

Chiba, Y., 2010. Bias analysis for the principal stratum direct effect in the presence of confounded intermediate variables. J. Biomet. Biostat., 1: 101101. DOI: $10.4172 / 2155-6180.1000101$

Chiba, Y., 2012. The large sample bounds on the principal strata effect with application to a prostate cancer prevention trial. Int. J. Biost. DOI: 10.1515/1557-4679.1365, PMID: 22628357

Chiba, Y., 2013. A simple method of measuring vaccine effects on infectiousness and contagion. Open $\mathrm{J}$. Stat., 3: 7-15. DOI: 10.4236/ojs.2013.34A002

Chiba, Y. and M. Taguri, 2013. Conditional and unconditional infectiousness effects in vaccine trials. Epidemiology, 24: 336-337. DOI: 10.1097/EDE.0b013e31828261f5

Chiba, Y. and T.J. VanderWeele, 2011. A simple method for principal strata effects when the outcome has been truncated due to death. Am. J. Epidemiol., 173: 745-751. DOI: 10.1093/aje/kwq418

Cole, S.R. and M.A. Hernán, 2008. Constructing inverse probability weights for marginal structural models. Am. J. Epidemiol., 168: 656-664. DOI: 10.1093/aje/kwn164

Frangakis, C.E. and D.B. Rubin, 2002. Principal stratification in causal inference. Biometrics, 58: 2129. DOI: $10.1111 / j .0006-341 X .2002 .00021 . x$

Gleason, D.F., 1966. Classification of prostatic carcinomas. Cancer Chemotherapy Rep., 50: 125128. PMID: 5948714

Lange, T., S. Vansteelandt and M. Bekaert, 2012. A simple unified approach for estimating natural direct and indirect effects. Am. J. Epidemiol., 176: 190195. DOI: $10.1093 / \mathrm{aje} / \mathrm{kwr} 525$

Lucia, M.S., J.I. Epstein, P.J. Goodman, A.K. Darke and V.E. Reuter et al., 2007. Finasteride and high-grade prostate cancer in the prostate cancer prevention trial. J. Nat. Cancer I., 99: 1375-1383. DOI: 10.1093/jnci/djm117

Manski, C.F., 1997. Monotone treatment response. Econometrica, 65: 1311-1334. DOI: $10.2307 / 2171738$ 
Pearl, J., 2001. Direct and indirect effects. Proceedings of the 17th Conference on Uncertainty in Artificial Intelligence, (UAI' 01), Morgan Kaufmann, San Francisco, pp. 411-420.

Phillips, C., 2008. Studies make case for finasteride to prevent prostate cancer. NCI Cancer Bull., 5: 1-4.

Redman, M.W., C.M. Tangen, P.J. Goodman, M.S. Lucia and C.A. Coltman, Jr. et al., 2008. Finasteride does not increase the risk of high-grade prostate cancer: A bias-adjusted modeling approach. Cancer Prev. Res., 1: 174-181. DOI: 10.1158/19406207.CAPR-08-0092

Robins, J.M., 2003. Semantics of Causal DAG Models and the Identification of Direct and Indirect Effects. In: Highly structured stochastic system, Green, P.J., N.L. Hjort and S. Richardson (Eds.), Oxford University Press, New York, pp: 70-82.

Robins, J.M., A. Rotnitzky, N. Jewell, K. Dietz and V. Farewell, 1992. Recovery of Information and Adjustment for Dependent censoring Using Surrogate Markers. In: AIDS Epidemiology-Methodological issues, Jewell, N.P., K. Dietz and V.T. Farewell (Eds.), Birkhäuser, Boston, pp: 297-331.

Rubin, D.B., 1974. Estimating causal effects of treatments in randomized and nonrandomized studies. J. Educ. Psychol., 66: 688-701. DOI: 10.1037/h0037350

Rubin, D.B., 1978. Bayesian inference for causal effects: The role of randomization. Ann. Stat., 6: 34-58. DOI: $10.1214 / \mathrm{aos} / 1176344064$

Rubin, D.B., 1990. Formal mode of statistical inference for causal effects. J. Stat. Plan. Infer., 25: 279-292. DOI: $10.1016 / 0378-3758(90) 90077-8$

Rubin, D.B., 2004. Direct and indirect causal effects via potential outcomes. Scand. J. Stat., 31: 161-170. DOI: $10.1111 / \mathrm{j} .1467-9469.2004 .02-123 . \mathrm{x}$

Shepherd, B.E., M.W. Redman and D.P. Ankerst, 2008. Does finasteride affect the severity of prostate cancer? A causal sensitivity analysis. J. Am. Stat. Assoc., 103: 1392-1404. DOI: 10.1198/016214508000000706
Shpitser, I. and T.J. VanderWeele, 2011. A complete graphical criterion for the adjustment formula in mediation analysis. Int. J. Biostat., 7: 1-24. DOI: 10.2202/1557-4679.1297

Thompson, I.M., C. Chi, D.P. Ankerst, P.J. Goodman and C.M. Tangen et al., 2006. Effect of finasteride on the sensitivity of PSA for detecting prostate cancer. J. Natl. Cancer I., 98: 1128-1133. DOI: 10.1093/jnci/djj307

Thompson, I.M., P.J. Goodman, C.M. Tangen, M.S. Lucia and G.J. Miller et al., 2003. The influence of finasteride on the development of prostate cancer. $\mathrm{N}$ Eng. J. Med., 349: 215-224. DOI: 10.1056/NEJMoa030660

Valeri, L. and T.J. VanderWeele, 2013. Mediation analysis allowing for exposure-mediator interactions and causal interpretation: theoretical assumptions and implementation with SAS and SPSS macros. Psychol. Methods, 18: 137-150. DOI: 10.1037/a0031034

van der Laan, M.J. and M.L. Petersen, 2008. Direct effect models. Int. J. Biostat. DOI: 10.2202/15574679.1064

VanderWeele, T.J., 2009. Concerning the consistency assumption in causal inference. Epidemiology, 20: 880-883. DOI: 10.1097/EDE.0b013e3181bd5638

VanderWeele, T.J. and E.J. Tchetgen Tchetgen, 2011. Bounding the infectiousness effect in vaccine trials. Epidemiology, 22: 686-693. DOI: 10.1097/EDE.0b013e31822708d5

VanderWeele, T.J., E.J. Tchetgen Tchetgen and M.E. Halloran, 2012. Components of the indirect effect in vaccine trials: identification of contagion and infectiousness effects. Epidemiology, 23: 751-761. DOI: 10.1097/EDE.0b013e31825fb7a0

Zhang, J.L. and D.B. Rubin, 2003. Estimation of causal effects via principal stratification when some outcomes are truncated by "death". J. Educ. Behav. Stat., 28: 353-368. DOI: $10.3102 / 10769986028004353$ 